\section{Avaliação da cobertura da atenção básica à saúde em Salvador, Bahia, Brasil (2000 a 2007)}

\author{
Evaluation of primary care coverage in Salvador, \\ Bahia State, Brazil (2000 to 2007)
}

1 Instituto de Saúde Coletiva,
Universidade Federal da
Bahia, Salvador, Brasil.
Correspondence
M. O. Cardoso
Instituto de Saúde Coletiva,
Universidade Federal da
Bahia.
Rua Basílio da Gama s/n,
Salvador, BA 42700-000,
Brasil.
cardosomarcelle@gmail.com

Abstract

In order to test a method for evaluating coverage of primary healthcare services in Salvador, Bahia State, Brazil (2000-2007), a study of spatial and temporal clusters was performed, with the city and its 12 health districts as the analytical units. The real and potential coverage rates were estimated for various primary care procedures. Estimation of coverage rates used both the Ministry of Health guideline and a second standard aimed at establishing an approximation to the population's health needs. The data were obtained from the Outpatient Information System of the Unified National Health System (SIA-SUS). Low coverage rates were observed, despite a slight increase in real coverage for medical consultations (7.8\%) and basic nursing care (66.7\%). Meanwhile, dental consultations showed a $40 \%$ reduction. The study discussed the possibilities for using the methodology to monitor coverage and its limitations given the deficiencies in the information systems.

Evaluation; Health Services Coverage; Family Health; Primary Health Care
Marcelle de Oliveira Cardoso 1

Ligia Maria Vieira-da-Silva ${ }^{1}$

\section{Introdução}

A temática da universalização da atenção à saúde tem sido relacionada com o reconhecimento da saúde como parte dos direitos de cidadania e tem marcos tanto no Plano Beveridge e na criação do Sistema Nacional de Saúde Inglês 1, como na adoção de sistemas de seguro social inspirados em Bismarck 2. Embora faça parte de diversas cartas de intenção e acordos internacionais, como a Declaração de Alma Ata e a resolução da 58a Assembleia Geral da Organização Mundial da Saúde (OMS), a sua implementação tem apresentado reveses, mesmo em países que historicamente conseguiram atingi-la, como os da Europa Central, Oriental e da antiga União Soviética 3 . Esses países passam por reformas em seus sistemas de saúde, com alterações nas fontes de financiamento e modalidades de organização da atenção, o que tem modificado as possibilidades de acesso e utilização dos seus serviços de saúde.

Ainda que em revisões recentes haja uma tentativa de distinção do que seria atenção universal à saúde (universal health care) e cobertura universal à saúde (universal health coverage) 4 , o conceito de cobertura e sua medição não são problematizados. Particularmente no que diz respeito à atenção primária à saúde, são escassos os estudos que incorporam a avaliação da cobertura em suas análises. Foram identificadas 
apenas publicações que incorporam a avaliação da cobertura real como indicador da adesão a programas específicos ou de utilização de serviços essenciais 5,6 .

A cobertura universal tem sido definida como sinônimo de acesso à atenção integral 4 . Entretanto, a superposição do conceito de cobertura ao de acesso, este último também polissêmico ?, não auxilia na delimitação do objeto a ser avaliado e, ao contrário, dificulta a sua operacionalização, que resulta em perda de especificidade. Cobertura e acesso são dois conceitos relacionados, porém distintos. Em revisão sobre o conceito de acesso, Travassos et al. 7 mostram a superposição do conceito de acesso, por alguns autores, ao de cobertura, efetividade, qualidade técnica e satisfação, considerando importante buscar apreender de forma independente cada uma das etapas do processo de utilização. A opção pela avaliação de cobertura situa-se nessa direção. A avaliação da cobertura refere-se à disponibilidade e distribuição social dos recursos, sendo apenas um dos componentes da avaliação dos serviços de saúde 8 . Tem sido definida na literatura sobre avaliação de programas sociais como a proporção da população-alvo que é beneficiada por uma determinada intervenção ${ }^{9}$. Ademais, a distinção feita por Soberón 10, entre a cobertura potencial, que corresponde à capacidade e possibilidade da oferta, e a cobertura real, que busca estimar a proporção da população que de fato utilizou os serviços, possui uma utilidade analítica que tem sido pouco explorada nos trabalhos revisados.

No Brasil, a criação do Sistema Único de Saúde (SUS) proporcionou a melhoria na utilização dos serviços com extensão da cobertura assistencial a grande contingente populacional que antes não tinha garantido o cuidado à saúde. Os dados da Pesquisa Nacional por Amostra de Domicílios (PNAD 2008) 11 revelaram que aproximadamente $95 \%$ das pessoas que procuraram atendimento de saúde tiveram sucesso e utilizaram o serviço, sendo que, dentre elas, $59 \%$ foram atendidas em serviços públicos, o que aponta para a contribuição do SUS na utilização desse tipo de serviço.

Alguns estudos nacionais que avaliam a cobertura têm apontado para a persistência de problemas relacionados à sua ampliação na atenção básica, mesmo em áreas desenvolvidas 12,13. Também têm sido relatadas dificuldades na expansão e implantação do Programa Saúde da Família (PSF) em grandes centros urbanos 14,15. A resistência corporativa de estruturas tradicionais às mudanças nos processos de trabalho, o sucateamento de estruturas físicas e de recursos humanos 16, além da existência de extensa rede privada que compete com a mudança no modelo de atenção, reforçando o modelo hegemônico, têm sido apontados como entraves na mudança do modelo assistencial. Além disso, unidades do PSF muitas vezes apresentam desempenho semelhante ao modelo tradicional no que se refere à acessibilidade e porta de entrada, revelando diversidade na operacionalização do referido programa 17 .

Embora a realização de inquéritos populacionais para a avaliação de cobertura tenha a vantagem de identificar tanto o padrão de utilização dos serviços, quanto a demanda reprimida 13 , existem exemplos de importantes investigações avaliativas que se apoiam em dados secundários $16,18,19$. Além disso, tendo em vista que os gestores dos serviços de saúde necessitam monitorar a cobertura assistencial com objetivo de obter informações para o processo cotidiano de tomada de decisão, e que os inquéritos populacionais não podem ser realizados rotineiramente, é necessário o desenvolvimento e aperfeiçoamento de abordagens, técnicas e métodos para a avaliação da cobertura apoiada em dados produzidos pelos sistemas de informação em saúde. Nessa perspectiva, há estudos que incorporam, na análise dos resultados, além dos dados dos inquéritos, dados de registros oficiais 12,20, enquanto outros usam apenas dados rotineiros produzidos pelos serviços 18,21. No que se refere à comparação entre a cobertura real e potencial, foram identificadas apenas duas investigações que utilizaram tal abordagem 20,21, ainda assim, estão limitadas a ações e serviços específicos, inexistindo estudo que tenha realizado esse tipo de análise para a atenção básica de um município como um todo. Além do mais, os padrões utilizados para a estimativa das coberturas não incorporam indicadores capazes de estabelecer uma aproximação com as necessidades de saúde populacionais. Com o objetivo de contribuir para a superação dessas lacunas, o presente estudo usou uma abordagem metodológica para a avaliação da cobertura da atenção básica à saúde que, além de incorporar uma estimativa das necessidades de saúde, explora as diferenças intraurbanas e compara coberturas potenciais com reais. A realização do estudo em Salvador, Bahia, justificou-se por ser uma das últimas capitais a ter sido habilitada à gestão plena do sistema municipal e para a qual inexistiam estimativas de cobertura da atenção básica.

\section{Metodologia}

Foi realizado um estudo de agregados espacial e temporal, tendo como unidades de análise o Município de Salvador e seus Distritos Sanitários. Foram estimadas as coberturas potenciais e reais 
de consultas médicas e ações odontológicas da rede própria municipal de Salvador para 2007, desagregadas por Distrito Sanitário, além da estimativa da cobertura real desses serviços realizados por todos os prestadores SUS em Salvador no período de 2000 a 2007, bem como a evolução das ações de enfermagem para o mesmo período.

O Município de Salvador, capital do Estado da Bahia, ocupa uma extensão territorial de $707 \mathrm{~km}^{2}$. A sua organização político-administrativa compreende 18 Regiões Administrativas e 12 Distritos Sanitários 22. Segundo estimativas do Instituto Brasileiro de Geografia e Estatística (IBGE), para o ano de 2007, a população residente no município foi de 2.892.625 habitantes, sendo 53\% composto por mulheres e $47 \%$ por homens ${ }^{23}$. Por mais que Salvador tenha um Índice de Desenvolvimento Humano (IDH) considerado satisfatório $(0,805)$, ocupando a 467a posição entre os 5.507 municípios do Brasil e 1a posição no estado, possui um cenário de desigualdades expresso no índice de Gini $(0,66)$ 22. No ano de 2006, o município, que era gestor apenas da atenção básica, foi habilitado à Gestão Plena do Sistema Municipal. Em 2007, estavam em funcionamento 419 estabelecimentos de saúde - incluindo unidades básicas, especializadas e serviços de emergência-, sendo $38 \%$ públicos, $6 \%$ filantrópicos e $56 \%$ privados lucrativos. No mesmo período, 22,3\% da população estavam cobertos pelo Programa Agente Comunitário de Saúde (PACS) e 13,7\% pelo PSF (Departamento de Atenção Básica, Secretaria de Assistência à Saúde, Ministério da Saúde. Evolução do credenciamento e implantação da Estratégia Saúde da Família. http://dab.saude. gov.br/historico_cobertura_sf.php, acessado em Nov/2007).

As seguintes variáveis foram selecionadas como critérios para a avaliação da cobertura e selecionados, para cada variável, procedimentos de atenção básica da tabela do Sistema de Informações Ambulatoriais do SUS (SIA/ SUS; Bloco 1) 24 , de acordo com a Portaria $n^{o}$. 1.230/99 GM do Ministério da Saúde: (a) consultas médicas nas especialidades básicas (procedimentos 02.011.02-6, 02.011.03-4, 02.012.00-6 a 02.012.15); (b) ações básicas de enfermagem (procedimentos 01.022.05-9 a 01.022.13-0, 01.023.00-4 a 01.023.04-7, 04.011.04-0 e 04.011.06-6, 04.012.00-3 a 04.012.03-8); (c) ações básicas em odontologia (procedimentos 03.010.00-7, 03.011.00-3, 03.011.01-1, 03.02.00-2, 03.021.00-9, 03.021.01-7, 03.022.00-5 a 03.022.08-0, 03.030.00-8 a 03.031.12-8, 03.040.00-3, 03.041.00-0 a 03.041.07-7).

Para cada um desses critérios foram selecionados respectivamente os seguintes indicadores e padrões: (a) cobertura e concentração de consultas médicas básicas por habitante por ano (padrão: 1,50 consultas/habitante/ano, de acordo com a Portaria $n^{\circ}$. 91/2007 GM do Ministério da Saúde); (b) concentração de ações básicas de enfermagem por habitante por ano (padrão: 2,3 a 6,4 procedimentos básicos de enfermagem/habitante/ano, de acordo com a Portaria $n^{\circ}$. 1.101/2002 GM do Ministério da Saúde); (c) cobertura e concentração de ações básicas de odontologia por habitante por ano (padrão: 2,8 ações básicas odontológicas/habitante/ano, sendo 0,3 para primeira consulta odontológica e 2,5 para procedimentos básicos, de acordo com a Portaria $n^{\circ}$. 91/2007 GM).

Para a avaliação das consultas médicas, foram considerados dois padrões, o primeiro é aquele proposto pela Portaria $n^{\circ}$. 91/2007 GM de 1,50 consulta por habitante por ano. O segundo padrão foi calculado especificamente para o presente estudo com o objetivo de estabelecer uma aproximação com as necessidades de saúde que têm sido objeto de consenso dos gestores. Com essa finalidade, foram utilizados os parâmetros de consultas médicas propostos pelas diretrizes para a programação pactuada e integrada da assistência à saúde na atenção básica 25 nas seguintes áreas: saúde da criança, saúde da mulher, saúde do idoso, saúde do adulto, hanseníase (multibacilar), tuberculose e urgência. Dessa forma, foram selecionadas 18 ações consideradas como prioritárias para a atenção básica para as quais o número mínimo de consultas médicas estava estabelecido nas referidas diretrizes; em seguida, utilizando-se dados da distribuição populacional de Salvador, chegou-se ao número de 2,27 consultas/habitante/ano necessárias em 2007.

O padrão de procedimentos-hora usado para o cálculo da cobertura potencial foi o disposto na Portaria $n^{o}$. 1.101/2002 GM, segundo a qual, o número de procedimentos por hora preconizado para os médicos é de quatro consultas e para dentistas três procedimentos.

Tendo em vista que as informações sobre a capacidade instalada de recursos humanos para anos anteriores não estavam registradas no Cadastro Nacional de Estabelecimentos de Saúde (CNES) ou na Secretaria Municipal de Saúde de Salvador (SMS), não foi possível realizar a estimativa da evolução histórica da cobertura potencial. Tanto o CNES como a SMS fazem atualizações periódicas sem armazenar dados anteriores no sistema de informação ou no relatório de gestão. Portanto, foi calculada a cobertura potencial dos prestadores municipais apenas para o ano 2007.

A cobertura real (CR) e a cobertura potencial (CP) para cada critério foram calculadas pelas seguintes fórmulas: 


$$
\begin{gathered}
\mathrm{CR}=\begin{array}{c}
\text { número de procedimentos realizados } \\
\text { em determinado ano x } 100
\end{array} \\
\begin{array}{c}
\text { população alvo x padrão de concentração } \\
\text { do procedimento por ano }
\end{array} \\
\frac{\begin{array}{c}
\text { (número de profissionais x carga horária } \\
\text { semanal x } 4 \text { semanas } \times 11 \text { meses } x \\
\text { procedimentos-hora) } \times 100
\end{array}}{\text { população alvo x padrão de concentração }} \\
\text { do procedimento por ano }
\end{gathered}
$$

As concentrações das consultas (CC) por habitante das ações de enfermagem e odontologia foram calculadas pela seguinte fórmula: $\mathrm{CC}=$ número de procedimentos realizados
em determinado ano

população alvo

Foi feita análise da evolução das coberturas por meio de visualização gráfica da tendência histórica, além do cálculo da diferença percentual entre o primeiro e último ano da série. Também foram utilizadas na análise as diferenças percentuais entre as coberturas reais e potenciais.

As informações sobre a produção ambulatorial das ações básicas em Salvador, para o período de 2000 a 2007, por tipo de prestador, foram obtidas do SIA-SUS disponibilizadas no site do Departamento de Informática do SUS (DATASUS; http://www2.datasus.gov.br/ DATASUS/index.php). Enquanto os dados desagregados por Distrito Sanitário, que servem de fonte para alimentar o SIA-SUS, foram fornecidos pela Assessoria Técnica da Secretaria $\mathrm{Mu}$ nicipal de Saúde de Salvador (ASTEC-SMS) 26 e analisados com o apoio do software TabWin (DATASUS. http://portal.saude.gov.br/portal/ se/datasus/area.cfm?id_area=732).

Os dados sobre o número de profissionais e carga horária por Distrito Sanitário foram obtidos da Coordenadoria de Recursos Humanos da Secretaria Municipal de Saúde do Município (CDRH-SMS) 27. Os dados sobre a população dos Distritos Sanitários entre 2000 e 2007 foram obtidos do IGBE 23 .

\section{Resultados}

O presente estudo revelou persistência de baixas coberturas assistenciais a despeito da ampliação discreta verificada na produção de alguns tipos de ações básicas de saúde entre 2000 e 2007. Considerando os procedimentos básicos realizados por todos os prestadores SUS, a cobertura real de consultas médicas aumentou em 7,8\%, passando de 51,24\% para 55,23\% (Figura 1), as ações básicas de enfermagem evoluíram $66,7 \%$, passando de 1,23 procedimento por habitante para 1,95 (Figu- ra 2), enquanto as primeiras consultas odontológicas foram reduzidas em $40 \%$, passando de 0,10 para 0,06 (Figura 2). Analisando-se a evolução da série temporal desse conjunto de prestadores, observou-se que as consultas médicas apresentaram expansão até 2003, quando se iniciou uma tendência de declínio (Figura 1). Com relação aos atendimentos odontológicos houve progressiva redução das primeiras consultas odontológicas. Já as ações de enfermagem foram as únicas com tendência clara de crescimento no período (Tabela 1 e Figura 2).

Contudo, a despeito dessa ampliação, verificou-se que havia desigualdade entre os Distritos Sanitários no que diz respeito à cobertura de todos os procedimentos investigados, realizados pela rede própria municipal (Tabela 1, Figuras 3 e 4). Os Distritos Sanitários Centro Histórico e Liberdade foram aqueles que apresentaram maiores coberturas reais para todas as ações consideradas, enquanto o Distrito de Pau da Lima foi aquele que obteve menor cobertura real de consultas médicas $(9,55 \%)$ e ações odontológicas $(2,67 \%)$ (Figuras 3 e 4 ).

A rede própria de serviços, sob gestão da SMS, estava composta, em 2007, por 98 unidades básicas de atendimento que correspondiam a $72,1 \%$ das unidades prestadoras desses serviços em Salvador. Desse modo, o governo municipal era o principal responsável pela realização de ações básicas no município, no período analisado. A análise da capacidade instalada de profissionais da rede revelou a existência de dois médicos, dois enfermeiros e um odontólogo para cada 10 mil habitantes em 2007. Esse quadro se refletiu tanto na cobertura potencial de consultas médicas básicas, no mesmo ano, que foi de $75,43 \%$, de acordo com o padrão ministerial de 1,50 consulta por habitante/ano, quanto na cobertura real que foi de $25,74 \%$ (Figura 3 ). Já a cobertura potencial para todos os procedimentos básicos de odontologia foi de $23,85 \%$, enquanto a real foi de $7,54 \%$ (Figura 4). Os Distritos Sanitários do Centro Histórico, da Liberdade e de Itapuã foram aqueles que apresentaram maiores coberturas potenciais para todas as ações consideradas. Esses três Distritos Sanitários apresentaram cobertura potencial de consultas médicas acima de $100 \%$, isto é, a quantidade de recursos humanos disponível superava a necessidade de consultas do local de acordo com o padrão ministerial. Já o Distrito Sanitário de Cajazeiras se destacou por estar, para todas as ações selecionadas, entre os que apresentaram as menores coberturas potenciais e reais. Também chamou a atenção o Distrito de Brotas, por ter apresentado a menor cobertura potencial para consultas médicas $(31,47 \%)$ (Figuras 3 e 4 ). 
Figura 1

Cobertura real (em \%) de consultas médicas básicas para todos os prestadores SUS, de acordo com o padrão ministerial * e as necessidades mínimas de consultas estimadas **. Salvador, Bahia, Brasil, 2000-2007.

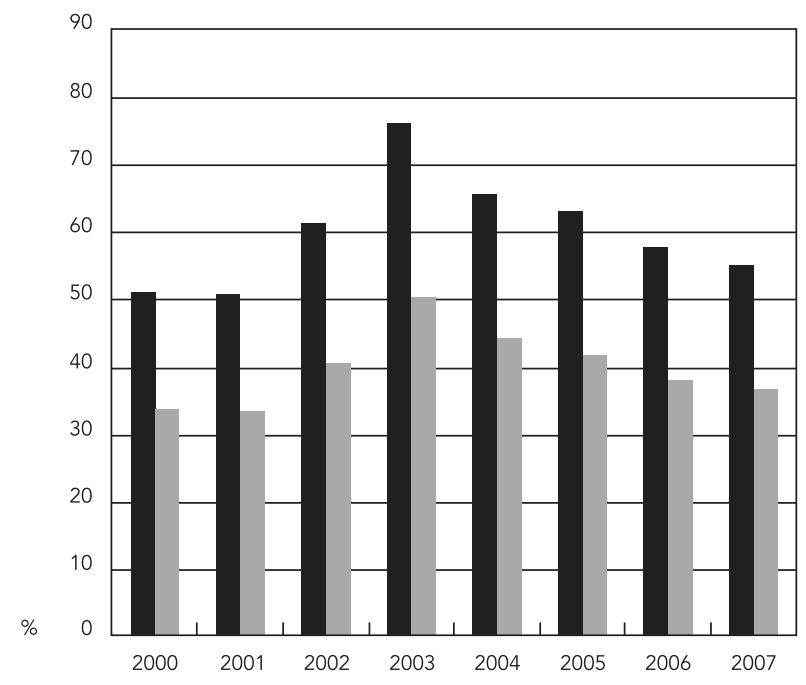

Cobertura real (padrão ministerial)

Cobertura real (necessidades mínimas estimadas)

* 1,50 consulta/habitante/ano;

** 2,27 consultas/habitante/ano.

Fonte: Sistema de Informações Ambulatoriais do SUS (SIA-SUS. Departamento de Informática do SUS;

http://www2.datasus.gov.br/DATASUS/index.php).

Figura 2

Concentração (por habitante/ano) de ações básicas de enfermagem e primeira consulta odontológica realizadas por todos os prestadores SUS. Salvador, Bahia, Brasil, 2000-2007.
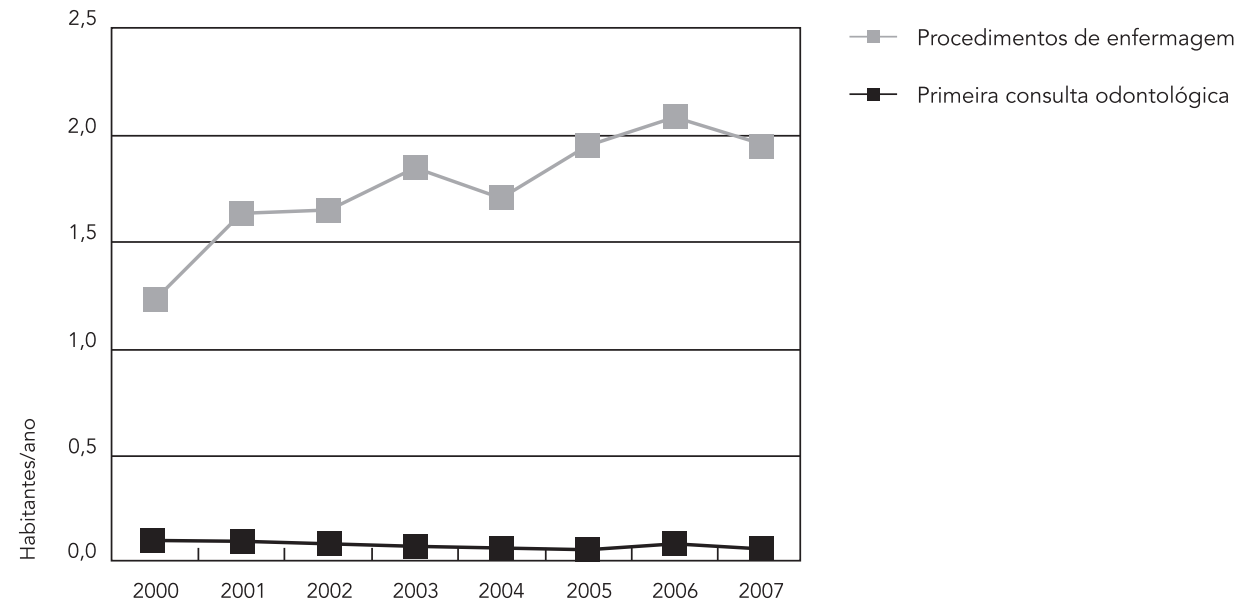

Fonte: Sistema de Informações Ambulatoriais do SUS (SIA-SUS. Departamento de Informática do SUS;

http://www2.datasus.gov.br/DATASUS/index.php). 
Tabela 1

Procedimentos da atenção básica, realizados e potenciais, segundo modalidades, local, ano, prestador e população. Salvador, Bahia, Brasil, $2000-2007$.

\begin{tabular}{|c|c|c|c|c|c|c|c|c|}
\hline \multirow[t]{3}{*}{ Local } & \multirow[t]{3}{*}{ Ano } & \multirow[t]{3}{*}{ Prestador * } & \multirow[t]{3}{*}{ População } & \multicolumn{5}{|c|}{ Modalidades de procedimentos da atenção básica } \\
\hline & & & & \multicolumn{2}{|c|}{ Médicos } & \multicolumn{2}{|c|}{ Odontológicos } & \multirow{2}{*}{$\begin{array}{c}\text { Enfermagem } \\
\text { Realizados }\end{array}$} \\
\hline & & & & Realizados ** & Potenciais *** & Realizados & Potenciais & \\
\hline Salvador & 2000 & Rede SUS & 2.443 .107 & 1.877 .772 & - & 488.621 & - & 3.005 .022 \\
\hline Salvador & 2001 & Rede SUS & 2.485 .702 & 1.892 .241 & - & 497.140 & - & 4.026 .837 \\
\hline Salvador & 2002 & Rede SUS & 2.520 .705 & 2.319 .679 & - & 655.383 & - & 4.159 .163 \\
\hline Salvador & 2003 & Rede SUS & 2.556 .430 & 2.924 .300 & - & 639.108 & - & 4.703 .831 \\
\hline Salvador & 2004 & Rede SUS & 2.592 .239 & 2.551 .930 & - & 622.137 & - & 4.432 .729 \\
\hline Salvador & 2005 & Rede SUS & 2.673 .557 & 2.526 .110 & - & 561.447 & - & 5.186 .701 \\
\hline Salvador & 2006 & Rede SUS & 2.714 .977 & 2.346 .147 & - & 678.744 & - & 5.674 .302 \\
\hline Salvador & 2007 & Rede SUS & 2.892 .625 & 2.396 .395 & - & 694.230 & - & 5.640 .619 \\
\hline Salvador & 2007 & $\begin{array}{c}\text { Rede própria } \\
\text { municipal }\end{array}$ & 2.892 .625 & 1.117 .021 & 3.273 .072 & 610.744 & 1.931 .820 & 2.673 .790 \\
\hline $\begin{array}{l}\text { Distrito Sanitário } \\
\text { da Barra }\end{array}$ & 2007 & $\begin{array}{c}\text { Rede própria } \\
\text { municipal }\end{array}$ & 356.457 & 160.204 & 283.712 & 73.626 & 233.640 & 418.493 \\
\hline $\begin{array}{l}\text { Distrito Sanitário } \\
\text { da Boca do Rio }\end{array}$ & 2007 & $\begin{array}{c}\text { Rede própria } \\
\text { municipal }\end{array}$ & 128.179 & 78.223 & 70.400 & 24.798 & 47.520 & 321.804 \\
\hline $\begin{array}{l}\text { Distrito Sanitário } \\
\text { de Brotas }\end{array}$ & 2007 & $\begin{array}{c}\text { Rede própria } \\
\text { municipal }\end{array}$ & 249.816 & 67.146 & 117.920 & 22.380 & 182.160 & 97.640 \\
\hline $\begin{array}{l}\text { Distrito Sanitário } \\
\text { de Cabula }\end{array}$ & 2007 & $\begin{array}{c}\text { Rede própria } \\
\text { municipal }\end{array}$ & 413.141 & 129.805 & 425.920 & 97.444 & 153.780 & 286.134 \\
\hline $\begin{array}{l}\text { Distrito Sanitário } \\
\text { de Cajazeiras }\end{array}$ & 2007 & $\begin{array}{c}\text { Rede própria } \\
\text { municipal }\end{array}$ & 183.203 & 40.853 & 140.800 & 21.460 & 87.120 & 107.611 \\
\hline $\begin{array}{l}\text { Distrito Sanitário } \\
\text { do Centro }\end{array}$ & 2007 & $\begin{array}{c}\text { Rede própria } \\
\text { municipal }\end{array}$ & 68.847 & 129.246 & 205.920 & 82.955 & 243.540 & 209.261 \\
\hline $\begin{array}{l}\text { Distrito Sanitário } \\
\text { de Itapagipe }\end{array}$ & 2007 & $\begin{array}{c}\text { Rede própria } \\
\text { municipal }\end{array}$ & 169.422 & 52.330 & 144.320 & 27.382 & 71.280 & 71.609 \\
\hline $\begin{array}{l}\text { Distrito Sanitário } \\
\text { de Itapuã }\end{array}$ & 2007 & $\begin{array}{c}\text { Rede própria } \\
\text { municipal }\end{array}$ & 216.903 & 84.343 & 396.000 & 47.499 & 170.280 & 258.770 \\
\hline $\begin{array}{l}\text { Distrito Sanitário } \\
\text { da Liberdade }\end{array}$ & 2007 & $\begin{array}{c}\text { Rede própria } \\
\text { municipal }\end{array}$ & 170.700 & 128.986 & 396.880 & 79.839 & 162.360 & 434.201 \\
\hline $\begin{array}{l}\text { Distrito Sanitário } \\
\text { de Pau da Lima }\end{array}$ & 2007 & $\begin{array}{c}\text { Rede própria } \\
\text { municipal }\end{array}$ & 337.055 & 48.275 & 413.600 & 25.186 & 97.020 & 187.408 \\
\hline $\begin{array}{l}\text { Distrito Sanitário } \\
\text { de São Caetano }\end{array}$ & 2007 & $\begin{array}{c}\text { Rede própria } \\
\text { municipal }\end{array}$ & 257.968 & 67.099 & 241.120 & 28.251 & 146.520 & 113.315 \\
\hline $\begin{array}{l}\text { Distrito Sanitário } \\
\text { do Subúrbio }\end{array}$ & 2007 & $\begin{array}{c}\text { Rede própria } \\
\text { municipal }\end{array}$ & 340.934 & 130.511 & 436.480 & 79.924 & 336.600 & 167.544 \\
\hline
\end{tabular}

* Rede SUS - procedimentos realizados por todos os prestadores SUS no município; rede própria municipal - procedimentos realizados por unidades básicas geridas pela Secretaria Municipal de Saúde de Salvador;

** Número total de procedimentos realizados no ano;

*** Número de consultas calculado com base na capacidade instalada do município: carga horária anual dos profissionais $x$ padrão de procedimentos/hora (4 consultas médicas; 2,8 procedimentos odontológicos e 3 procedimentos de enfermagem).

Fonte: Sistema de Informações Ambulatoriais do SUS (SIA-SUS. Departamento de Informática do SUS; http://www2.datasus.gov.br/DATASUS/index.php); Assessoria Técnica de Planejamento, Secretaria Municipal de Saúde de Salvador 25 e Coordenadoria de Recursos Humanos, Secretaria Municipal de Saúde de Salvador 26 . 
Cobertura (em \%) potencial e real de consultas médicas nas especialidades básicas para prestador municipal, por Distrito Sanitário * e diferença percentual. Salvador, Bahia, Brasil, 2007.

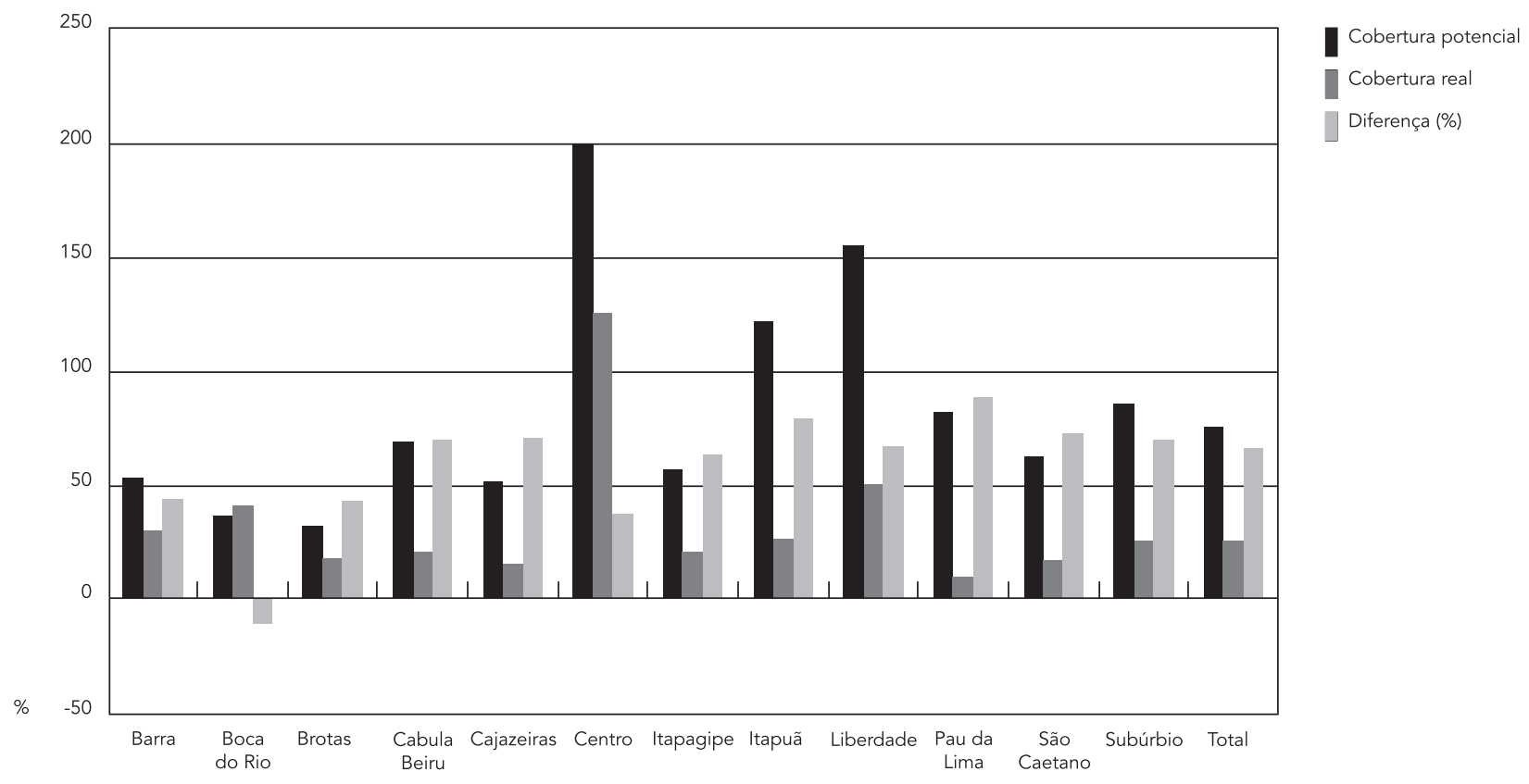

Fonte: Assessoria Técnica de Planejamento, Secretaria Municipal de Saúde de Salvador 25 e Coordenadoria de Recursos Humanos, Secretaria Municipal de Saúde de Salvador 26.

* Considerando o padrão ministerial de 1,50 consulta/habitante/ano (Portaria n. 91/2007 GM do Ministério da Saúde).

Comparando-se as coberturas potencial e real, encontrou-se que nem sempre maior cobertura potencial implicou maior cobertura real, ou seja, em melhor utilização da capacidade disponível. Em todos os Distritos Sanitários, foram observadas diferenças percentuais entre as coberturas potencial e real de consultas médicas e ações de odontologia que variaram entre -11,09\% e $88,33 \%$ (Figuras 3 e 4). O Distrito da Boca do Rio apresentou a menor diferença entre as coberturas potencial e real, tendo sido a produção de consultas médicas, em 2007, superior à capacidade instalada.

Quando comparados os dois padrões utilizados para cálculo das coberturas reais de consultas médicas, observou-se que tanto o padrão calculado de 2,27 consultas/habitante/ano, quanto o padrão de 1,50 da portaria ministerial não foram alcançados no período de 2000 a 2007. A cobertura real de consultas médicas básicas, de acordo com o padrão de 2,27 consultas/habitante/ano, foi de 33,85\%, em 2000, e 36,51\% em 2007 e de $51,24 \%$, em 2000, e 55,23\%, em 2007 quando utilizado o padrão ministerial de 1,50 consulta/ habitante/ano (Figura 1).

\section{Discussão}

O presente estudo reuniu evidências que apontam para insuficiências na atenção básica de saúde em Salvador, expressas nas baixas coberturas potencial e real, de algumas ações de saúde prioritárias. Apenas as ações de enfermagem apresentaram clara expansão, enquanto as ações médicas e odontológicas demonstraram grande variação, com tendência ao declínio, no período aqui analisado.

A variabilidade encontrada no desempenho dos Distritos Sanitários é consistente com a implantação do projeto para melhoria da acessibilidade e humanização do acolhimento elaborado em 2005 pela SMS 28 que atingiu parcialmente seus objetivos centrais, que eram a extinção das filas evitáveis e introdução de ações voltadas para a redução de obstáculos dos serviços conforme 
Cobertura (em \%) potencial e real de ações básicas odontológicas para prestador municipal, por Distrito Sanitário * e diferenças percentuais. Salvador, Bahia, Brasil, 2007.

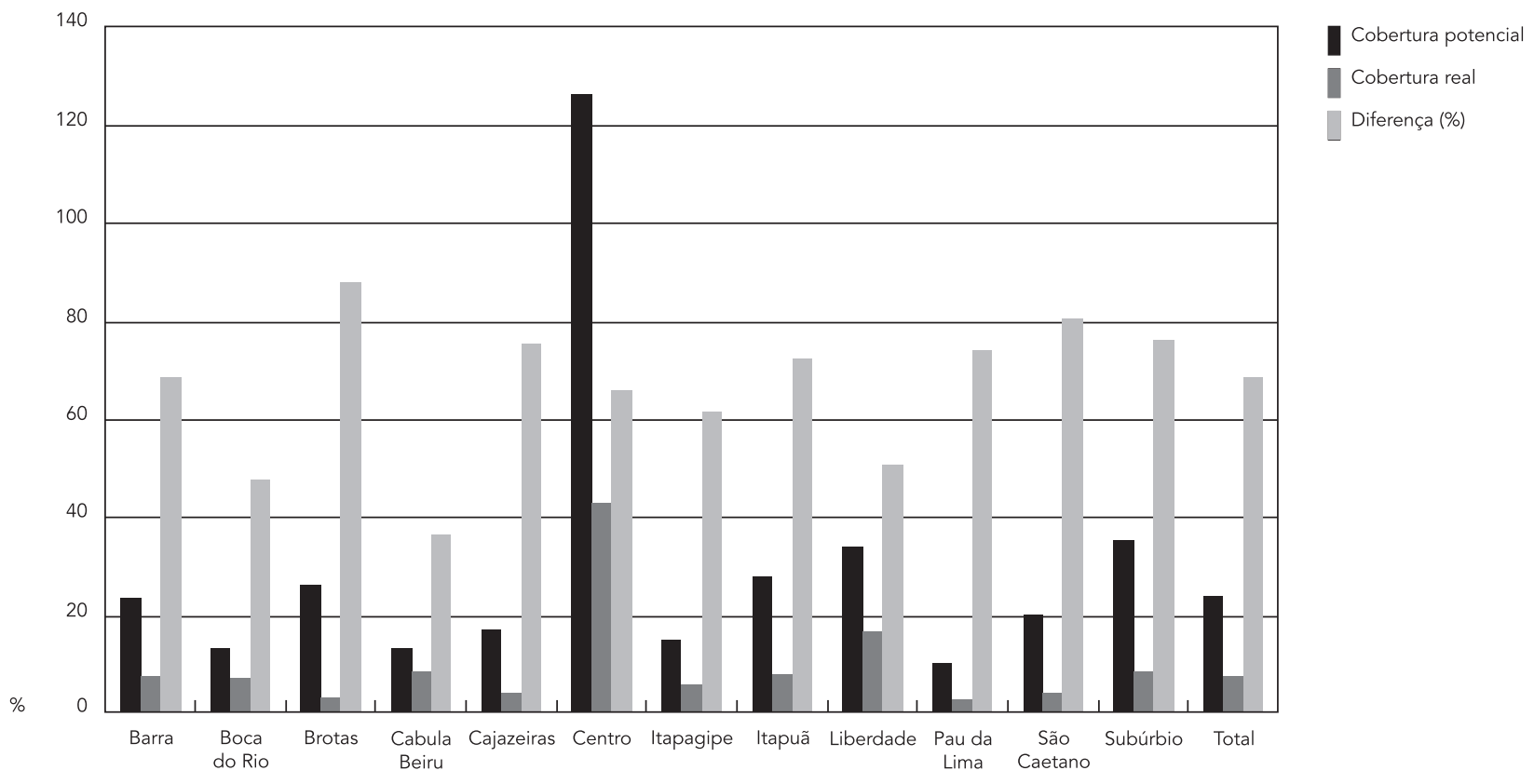

Fonte: Assessoria Técnica de Planejamento, Secretaria Municipal de Saúde de Salvador 25 e Coordenadoria de Recursos Humanos, Secretaria Municipal de Saúde de Salvador 26 .

* Considerando o padrão ministerial de 1,50 consulta/habitante/ano (Portaria n. 91/2007 GM do Ministério da Saúde).

ficou evidenciado em pesquisa avaliativa realizada em 2006 29. Ademais, problemas na gestão municipal da saúde, sobretudo no que diz respeito à autonomia na administração dos recursos e instabilidade dos quadros dirigentes, também podem estar relacionados com a explicação da situação analisada 30 .

As diferenças encontradas entre a cobertura potencial e real, em diversos Distritos Sanitários, podem ser indicativas da existência de má distribuição de profissionais, bem como da subutilização da carga horária dos mesmos, o que traduz problemas na gestão dos recursos disponíveis. Já o achado específico do Distrito da Boca do Rio de uma cobertura real superior àquela potencial, pode ser indicativo tanto de subestimação da capacidade instalada de profissionais, como de problemas no sistema de informação no que se refere ao registro dos procedimentos realizados. Considerando a média do município, é pouco provável que a cobertura real tenha sido superior a potencial. Da mesma forma, a cobertura real superior a $100 \%$, como a encontrada no Distrito do Centro Histórico, pode traduzir subestimação da população ou atendimento a usuários oriundos de outros Distritos Sanitários, o que é dissonante do modelo assistencial de base territorial, previsto no Plano Municipal de Saúde de Salvador 22. Já as coberturas potenciais superiores a $100 \%$, como as encontrada nos Distritos do Centro Histórico, de Itapuã e da Liberdade possivelmente indicam que a distribuição dos recursos humanos no município não tem seguido uma lógica territorial.

As baixas coberturas encontradas traduzem a combinação entre crescimento insuficiente da oferta de serviços frente ao aumento populacional, que, por sua vez, gera aumento na demanda assistencial. Decorrem da pequena expansão verificada na rede de atendimento do município como um todo, e, do PSF em particular, aliada à insuficiente contratação de pessoal. De fato, verificou-se que entre 2004 e 2007 ocorreu a criação de apenas 34 novas equipes e 12 novas unidades do PSF, o que correspondia a 40,9\% das unidades básicas municipais. Esse incremento correspon- 
dia a uma cobertura potencial do PSF de apenas 13,7\%, para o município, em dezembro de 2007. Tal cobertura do PSF pode ser considerada baixa especialmente quando comparada à de outras capitais brasileiras de equivalente porte populacional como Fortaleza (Ceará) e Belo Horizonte (Minas Gerais) que apresentavam no mesmo período coberturas do PSF de $35,1 \%$ e $69,6 \%$, respectivamente (Departamento de Atenção Básica, Secretaria de Assistência à Saúde, Ministério da Saúde. Evolução do credenciamento e implantação da Estratégia Saúde da Família. http://dab. saude.gov.br/historico_cobertura_sf.php, acessado em Nov/2007).

No caso específico de Salvador, essa situação estrutural era agravada pela existência de barreiras organizacionais, a exemplo do sistema de agendamento de consultas, que por ocorrer de forma periódica (semanal ou mensal), em grande parte das unidades, resultavam na formação de longas filas e grandes períodos de espera entre a marcação e a consulta ${ }^{28}$. Além disso, o descumprimento das cargas horárias contratuais pelos profissionais, em razão da existência de múltiplos vínculos de trabalho, resultava na redução no número de consultas ofertadas e na duração do atendimento; gerentes de unidades pouco capacitados para exercer a função; baixa remuneração e irregular abastecimento de insumos na rede também foram relatados pelos gerentes de unidades básicas de saúde em diagnóstico que precedeu a elaboração do projeto para melhoria da acessibilidade e humanização do acolhimento elaborado em 2005 pela SMS 28 .

A insuficiente cobertura assistencial observada relaciona-se com as circunstâncias históricas de desenvolvimento do SUS municipal e problemas decorrentes da institucionalização do SUS no país como um todo. A natureza do Estado brasileiro - patrimonialista, autoritário, burocrático, colonizado por interesses privados - ao lado da descontinuidade administrativa, decorrente da constante mudança na correlação de forças políticas no âmbito institucional 31, podem explicar a incipiência na implantação do SUS municipal e, em particular, o atraso na adoção da gestão plena do sistema.

Problemas semelhantes têm sido encontrados e explicam as dificuldades de expansão e implantação do PSF nas grandes cidades do Brasil 14,15, tais como dificuldade na mudança dos processos de trabalho e insuficiência da estrutura física e de recursos humanos 16. Todavia, investigações adicionais são necessárias para uma melhor compreensão dos diversos fatores relacionados à gestão de recursos humanos, com o desempenho das unidades de saúde e fluxos de demanda que implicam a variabilida- de no uso e baixo aproveitamento dos recursos disponíveis.

O padrão encontrado, específico para o $\mathrm{Mu}$ nicípio de Salvador, de 2,27 consultas médicas por habitante por ano, calculado com base nas necessidades estimadas de consultas médicas para 18 ações priorizadas pelas diretrizes da programação pactuada e integrada (PPI), mostra que o padrão nacional proposto pela Portaria $n^{\circ}$. $91 / 2007$ GM, de 1,50 consulta por habitante por ano é insuficiente para avaliar as necessidades populacionais.

A metodologia deste estudo se baseou na utilização de dados secundários do SIA-SUS que se revelou importante ferramenta para o monitoramento da cobertura de algumas ações básicas de saúde. Seu principal mérito se relaciona à disponibilidade dos dados para os gestores $\mathrm{e}$ à simplicidade das técnicas utilizadas. Diversos estudos com dados secundários dos sistemas de informação em saúde têm revelado a potencialidade dos mesmos, como geradores de informações para a gestão dos serviços 32,33,34. Contudo, ainda que tenham sido registradas melhorias na qualidade dos sistemas de informação em saúde 35 , e em particular do SIA-SUS, persistem problemas, havendo ainda a necessidade da realização de estudos que validem as informações disponíveis.

Com relação aos padrões utilizados para estimar as coberturas, recomenda-se o aperfeiçoamento do indicador das necessidades de consultas, por meio do uso de estudos epidemiológicos que produzam informações sobre a situação de saúde do território. Em particular, o valor médio de consultas necessárias para o tratamento de cada agravo deve ser continuamente renovado, apoiado em consensos de especialistas. Além disso, precisam ser aperfeiçoados os padrões para cálculo da cobertura potencial da atenção básica à saúde de forma que seja possível subsidiar a gestão da carga horária das equipes de profissionais, sem deixar de incluir indicadores adicionais que permitam aferir além da produtividade a qualidade do cuidado.

\section{Considerações finais}

O presente estudo revelou a importância do uso de séries históricas de indicadores de cobertura como ferramenta para a avaliação da adequação e disponibilidade de recursos da atenção básica, permitindo o acompanhamento da evolução das ações de saúde, ao longo do tempo, no âmbito de um determinado território. As baixas coberturas aqui encontradas, quando comparadas com outras capitais, revelam a heterogeneidade 
na implantação do SUS no país e apontam para a persistência de obstáculos. As principais limitações do estudo estão relacionadas ao fato de que os indicadores aqui utilizados não são sensíveis para captar mudanças no modelo assistencial e no processo de trabalho, como a organização da oferta e qualidade do cuidado, o que implica que a avaliação da cobertura precisa ser complementada por outros estudos avaliativos referentes à acessibilidade, à qualidade técnica e à efetividade.

Mesmo que a avaliação da cobertura assistencial seja uma das mais simples e elementares aferições, com as limitações aqui colocadas,

\section{Resumo}

Com o objetivo de testar abordagem metodológica para avaliação da cobertura dos serviços de atenção básica em Salvador, Bahia, Brasil (2000 a 2007), foi realizado um estudo de agregados espacial e temporal, tendo como unidades de análise o município e seus 12 Distritos Sanitários. Foram estimadas as coberturas real e potencial de diversos procedimentos realizados na atenção básica. Para a estimativa das coberturas, além do padrão ministerial, foi elaborado um segundo padrão com o objetivo de estabelecer uma aproximação com as necessidades de saúde da população. Os dados foram obtidos do Sistema de Informações Ambulatoriais do Sistema Único de Saúde (SIA-SUS). Foram encontradas baixas coberturas assistenciais no período analisado, a despeito da ampliação discreta verificada tanto na cobertura real de consultas médicas $(7,8 \%)$, quanto na produção de ações básicas de enfermagem (66,7\%). Já as consultas odontológicas apresentaram redução em 40\%. Foram discutidas tanto as possibilidades de uso da metodologia para o monitoramento da cobertura, como as suas limitações tendo em vista as deficiências dos sistemas de informação.

Avaliação; Cobertura de Serviços de Saúde; Saúde da Família, Atenção Básica à Saúde possui o mérito de permitir que o gestor problematize a atenção à saúde com base em um território e uma população - com seus problemas e necessidades de saúde -, sendo complementar e auxiliar para o acompanhamento de indicadores de resultados. Permite, além do mais, aferir a disponibilidade e distribuição dos recursos, na perspectiva de um sistema de saúde que tem como diretriz a universalização da atenção, como é o caso do SUS brasileiro. A metodologia aqui utilizada poderá ser adaptada e utilizada para o monitoramento de ações prioritárias dos serviços de saúde em diversos níveis.

\section{Colaboradores}

M. O. Cardoso contribuiu na concepção, planejamento, análise e interpretação dos dados, na elaboração do rascunho e na aprovação da versão final do manuscrito. L. M. Vieira-da-Silva colaborou na concepção, planejamento, análise e interpretação dos dados, elaboração da revisão crítica do conteúdo e na aprovação da versão final do manuscrito. 


\section{Referências}

1. Fiori JL. Estado de bem-estar social: padrões e crises. Physis (Rio J.) 1997; 7:129-47.

2. Garret L, Chowdhury AMR, Pablos-Méndez A. All for universal health coverage. Lancet 2009; 374:1294-9.

3. Mills A. Strategies to achieve universal coverage: are there lessons from middle income countries? A literature review commissioned by the Health Systems Knowledge Network. Health Economics and Financing Programme. Geneva: World Health Organization; 2007.

4. Stuckler D, Feigl AB, Basu S, McKee M. The political economy of universal health coverage. Background paper for the Global Symposium on Health System Research. Geneva: World Health Organization; 2010.

5. Akinleye SO, Falade CO, Ajayi IO. Knowledge and utilization of intermittent preventive treatment for malaria among pregnant women attending antenatal clinics in primary health care centers in rural southwest, Nigeria: a cross-sectional study. BMC Pregnancy Childbirth 2009; 9:28.

6. Cooke GS, Little KE, Bland RM, Thulare H, Newell ML. Need for timely paediatric HIV treatment within primary health care in rural South Africa. PLoS One 2009; 4:e7101.

7. Travassos C, Martins M. Uma revisão sobre os conceitos de acesso e utilização de serviços de saúde. Cad Saúde Pública 2004; 20 Suppl 2:S190-8.

8. Vieira-da-Silva LM. Conceitos, abordagens e estratégias para a avaliação em saúde. In: Vieira-daSilva LM, Hartz ZMA, organizadoras. Avaliação em saúde: dos modelos teóricos à prática na avaliação de programas e sistemas de saúde. Rio de Janeiro: Editora Fiocruz/Salvador: Edufba; 2005. p. 15-39.

9. Rossi PH, Lipsey MW, Freeman HE. Assessing and monitoring program process. In: Rossi PH, Lipsey MW, Freeman HE, editors. Evaluation, a systematic approach. Beverly Hills: Sage Publications; 2004. p. 169-201.

10. Soberón G. La extensión de cobertura de los servicios de salud. Gac Méd Méx 1988; 124:163-75.

11. Instituto Brasileiro de Geografia e Estatística. Pesquisa Nacional por Amostra de Domicílios: um panorama da saúde no brasil. Acesso e utilização dos serviços, condições de saúde e fatores de risco e de proteção à saúde. Rio de Janeiro: Instituto Brasileiro de Geografia e Estatística; 2008.

12. Pessoto UC, Heimann LS, Boaretto RC. Desigualdades no acesso e utilização dos serviços de saúde na Região Metropolitana de São Paulo. Ciênc Saúde Coletiva 2007; 12:351-62.

13. César CLG, Tanaka OY. Inquérito domiciliar como instrumento de avaliação de serviços de saúde: um estudo de caso na região sudoeste da área metropolitana de São Paulo, 1989-1990. Cad Saúde Pública 1996; 12 Suppl 2:59-70.
14. Facchini LA, Piccini RX, Tomasi E, Silveira DS, Teixeira VA, Maia MFS, et al. Contribuições do Estudo de Linha de Base da UFPEL às políticas, aos serviços e à pesquisa em atenção básica à saúde. In: Hartz Z, Felisberto E, Silva LV, organizadores. Meta-avaliação da atenção básica em saúde: teoria e prática. Rio de Janeiro: Editora Fiocruz; 2008. p. 167-97.

15. Viana AL, Rocha JSY, Elias PE, Ibañez N, Novaes MHD. Modelos de atenção básica nos grandes municípios paulistas: efetividade, eficácia, sustentabilidade e governabilidade. Ciênc Saúde Coletiva 2006; 11:577-603.

16. Ronzani TM, Stralen CJ. Dificuldades de implantação do Programa Saúde da Família como estratégia de reforma do sistema de saúde brasileiro. Revista de Atenção Primária à Saúde 2003; 2:99-107.

17. Cordeiro H, Pereira T, Costa H, Pereira ATS. Acreditação como avaliação nos serviços de atenção primária em saúde. In: Hartz Z, Felisberto E, Vieira-da-Silva LM, organizadores. Meta-avaliação da atenção básica da saúde; teoria e prática. Rio de Janeiro: Editora Fiocruz; 2008. p. 153-66.

18. Nascimento ER, Paiva MS, Rodrigues QP. Avaliação da cobertura e indicadores do Programa de Humanização do Pré-natal e Nascimento no município de Salvador, Bahia, Brasil. Rev Bras Saúde Matern Infant 2007; 7:191-7.

19. Aquino R, Oliveira NF, Barreto ML. Impact of the Family Health Program in infant mortality in Brazilian municipalities. Am J Public Health 2009; 99:87-93.

20. Cavalcante MGS, Samico I, Frias PG, Vidal SA. Análise de implantação das áreas estratégicas da atenção básica nas equipes de saúde da família em município de uma Região Metropolitana do Nordeste Brasileiro. Rev Bras Saúde Matern Infant 2006; 6:437-45.

21. Ribeiro-Sobrinho C, Souza LEPF, Chaves SCL. Avaliação da cobertura do Serviço Odontológico da Polícia Militar da Bahia em Salvador, Bahia, Brasil. Cad Saúde Pública 2008; 24:295-302.

22. Secretaria Municipal de Saúde de Salvador. Plano municipal de saúde 2006-2009. Salvador: Secretaria Municipal de Saúde de Salvador; 2005.

23. Instituto Brasileiro de Geografia e Estatística. Censo demográfico 2000 e estimativa populacional 2001-2007. Rio de Janeiro: Instituto Brasileiro de Geografia e Estatística; 2008.

24. Ministério da Saúde. Manual de preenchimento de instrumentação do SIA-SUS. http://dtr2001.saude.gov.br/sas/decas/indice.mansia.htm (acessado em Nov/2007).

25. Ministério da Saúde. Diretrizes operacionais: Pacto pela Vida, em Defesa do SUS e de Gestão. Brasília: Ministério da Saúde; 2006. (Série Pactos pela Saúde, 1). 
26. Assessoria Técnica de Planejamento, Secretaria Municipal de Saúde de Salvador. Banco de dados local do SAI-SUS: microdados por Distrito Sanitário [CD-ROM]. Salvador: Secretaria Municipal de Saúde de Salvador; 2007.

27. Coordenadoria de Recursos Humanos, Secretaria Municipal de Saúde de Salvador. Relatório técnico. Salvador: Secretaria Municipal de Saúde de Salvador; 2007.

28. Vieira-da-Silva LM, Esperidião MA, Viana SV, Alves VS, Lemos DVS, Caputo MC, et al. Avaliação da implantação de programa voltado para melhoria da acessibilidade e humanização do acolhimento aos usuários na rede básica: Salvador, 2005-2008. Rev Bras Saúde Matern Infant 2010; 10:131-43.

29. Vieira-da-Silva LM, Chaves SC, Esperidião MA, Lopes-Martinho RM. Accessibility to primary healthcare in the capital city of a northeastern state of Brazil: an evaluation of the results of a programme. J Epidemiol Community Health 2010; 64:1100-5.

30. Souza LEPF. O SUS necessário e o SUS possível: estratégias de gestão: uma reflexão a partir de uma experiência concreta. Ciênc Saúde Coletiva 2009; 14:911-8.
31. Paim JS, Teixeira CF. Configuração institucional e gestão do Sistema Único de Saúde: problemas e desafios. Ciênc Saúde Coletiva 2007; 12 Suppl:1819-29.

32. Silva AS, Laprega MR. Avaliação crítica do Sistema de Informação da Atenção Básica (SIAB) e de sua implantação na região de Ribeirão Preto, São Paulo, Brasil. Cad Saúde Pública 2005; 21:1821-8.

33. Medeiros KR, Machado HOP, Albuquerque PC, Gurgel Junior GD. O sistema de informação em saúde como instrumento da política de recursos humanos: um mecanismo importante na detecção das necessidades da força de trabalho para o SUS. Ciênc Saúde Coletiva 2005; 10:433-40.

34. Scochi MJ. Indicadores da qualidade dos registros e da assistência ambulatorial em Maringá, (Estado do Paraná, Brasil), 1991: um exercício de avaliação. Cad Saúde Pública 1994; 10:356-67.

35. Mota ELA, Carvalho DMT. Sistemas de informação em saúde. In: Rouquayrol MZ, Almeida Filho N, organizadores. Epidemiologia \& saúde. 6a Ed. Rio de Janeiro: Editora Medsi; 2003. p. 605-28.

Recebido em 02/Mai/2011

Versão final reapresentada em 23/Dez/2011 Aprovado em 13/Mar/2012 